\title{
Frenkel versus charge-transfer exciton dispersion in molecular crystals
}

\author{
Pierluigi Cudazzo, ${ }^{1,2, *}$ Matteo Gatti, ${ }^{1,2,3,4}$ Angel Rubio, ${ }^{1,2,5}$ and Francesco Sottile ${ }^{2,3}$ \\ ${ }^{1}$ Nano-Bio Spectroscopy Group, Departamento Física de Materiales, Universidad del País Vasco, Centro de Física de Materiales \\ CSIC-UPVIEHU-MPC and DIPC, Avenida Tolosa 72, E-20018 San Sebastián, Spain \\ ${ }^{2}$ European Theoretical Spectroscopy Facility (ETSF) \\ ${ }^{3}$ Laboratoire des Solides Irradiés, École Polytechnique, CNRS-CEA/DSM, F-91128 Palaiseau, France \\ ${ }^{4}$ Synchrotron SOLEIL, L'Orme des Merisiers, B.P. 48 Saint-Aubin, 91192 Gif-sur-Yvette, France \\ ${ }^{5}$ Theory Department, Fritz-Haber-Institut der Max-Planck-Gesellschaft, Faradayweg 4-6, D-14195 Berlin-Dahlem, Germany
}

(Received 26 June 2013; revised manuscript received 3 October 2013; published 27 November 2013)

\begin{abstract}
By solving the many-body Bethe-Salpeter equation at finite momentum transfer, we characterize the exciton dispersion in two prototypical molecular crystals, picene and pentacene, in which localized Frenkel excitons compete with delocalized charge-transfer excitons. We explain the exciton dispersion on the basis of the interplay between electron and hole hopping and electron-hole exchange interaction, unraveling a simple microscopic description to distinguish Frenkel and charge-transfer excitons. This analysis is general and can be applied to other systems in which the electron wave functions are strongly localized, as in strongly correlated insulators.
\end{abstract}

DOI: 10.1103/PhysRevB.88.195152

PACS number(s): 71.35.-y, 71.15.Qe, 78.40.Me

Excitons are neutral electronic excitations that dominate the low-energy part of the optical spectra in insulators and semiconductors. They consist of bound electron-hole (e-h) pairs that can be excited in several ways: by absorption of light and by relaxation of free electrons and holes after optical or electrical pumping. They play an essential role in many semiconductor applications (e.g., for light-emitting diodes, lasers, and photovoltaic cells) and give rise to the rich field of Bose-Einstein exciton condensates. ${ }^{1-3}$ In all these cases it is fundamental to understand the decay rate and the propagation of the excitons. The latter is directly related to their energy dispersion as a function of momentum transfer. Recent advances in loss spectroscopies make it possible to map out the full momentum-energy exciton dispersion. ${ }^{4-7}$ On the other hand, the interpretation of these experimental spectra requires first-principles theoretical approaches able to describe and analyze excitons at finite momentum transfer. The Bethe-Salpeter equation (BSE) from many-body perturbation theory has become the most accurate framework to describe excitonic effects in the optical spectra of many materials. ${ }^{8,9}$ However optical spectroscopy probes the zero-momentum-transfer limit only. Therefore first-principles analysis of the exciton dispersion is still an important goal to reach.

Molecular crystals represent a textbook case ${ }^{10,11}$ that clearly illustrates the need for advanced theoretical tools to understand the exciton dispersion. Typically, the lowest-energy excited states in these materials are strongly localized Frenkel (FR) excitons, where the interacting e-h pairs are localized on the same molecular unit. Charge-transfer (CT) excitons, in which e-h pairs are delocalized on different units, usually appear at higher energies in the spectra. However, when the molecular units are large enough, the effective interactions for e-h pairs localized on the same site or on two different sites become comparable and either CT or FR excitons can occur. Under these conditions many-body effects become crucial to set the character of the excitons and an ab initio treatment of the e-h interactions is thus required.
In the present work we solve the BSE at finite-momentum transfer ${ }^{12-14}$ to investigate two prototypical isoelectronic molecular crystals: picene and pentacene (see Fig. 1). By switching on the different e-h interactions step by step and analyzing their effects, we are able to draw a general picture of the exciton dispersion. We show that while the dispersion of FR excitons is set by the exchange e-h interaction, the dispersion of CT states is mainly related to the electronic band structure. Our conclusions have a general validity that holds in all systems in which the electron wave functions are strongly localized.

The BSE excitonic Hamiltonian in the basis of wave functions localized on the molecular units can be written as

$$
\begin{aligned}
\hat{H}_{\mathrm{ex}}= & \sum_{\mathbf{R} i, \mathbf{S} j} h_{\mathbf{R} i, \mathbf{S} j}^{e} a_{\mathbf{R} i}^{\dagger} a_{\mathbf{S}_{j}}-\sum_{\mathbf{R} i, \mathbf{S} j} h_{\mathbf{R} i, \mathbf{S} j}^{h} b_{\mathbf{R} i}^{\dagger} b_{\mathbf{S}_{j}} \\
& +\sum_{\mathbf{R} i, \mathbf{S} j, \mathbf{P} l, \mathbf{Q} m}\left(2 \bar{v}_{\mathbf{Q} m, \mathbf{R} i}^{\mathbf{S} j, \mathbf{P} l}-W_{\mathbf{Q} m \mathbf{R} i}^{\mathbf{S} j, \mathbf{P} l}\right) a_{\mathbf{R} i}^{\dagger} b_{\mathbf{Q} m}^{\dagger} b_{\mathbf{S} j} a_{\mathbf{P} l} .
\end{aligned}
$$

Here $a^{\dagger}(a)$ and $b^{\dagger}(b)$ are creation (annihilation) operators for electrons and holes, and the boldface and italic letters indicate the lattice vector and the molecular unit in the primitive cell, respectively. $h^{e}$ and $h^{h}$ are the one-particle Hamiltonians for the motion of free electrons and holes, which are described by the band structure calculated in the $G W$ approximation. ${ }^{15}$ The BSE kernel is given by the sum of $2 \bar{v}$, which includes only the $\mathbf{G} \neq 0$ reciprocal-space components of the bare Coulomb interaction $v$ and the statically screened Coulomb interaction $W$. The matrix elements of $\bar{v}$ and $W$ enter the BSE kernel as exchange and direct e-h interactions, respectively.

Due to the strong localization of the electronic wave functions on the molecular units, it is possible to neglect the overlap integrals between wave functions localized on different molecules. Under this condition the excitonic Hamiltonian $\hat{H}_{\text {ex }}$, Eq. (1), takes a simple block form, being the sum of three terms: $\hat{H}_{\mathrm{FR}}$ and $\hat{H}_{\mathrm{CT}}$, which describe FR and CT excitons, respectively, plus a coupling term, $\hat{H}_{\mathrm{CT}-\mathrm{FR}}$, which originates from hopping processes of independent e-h pairs and is related 

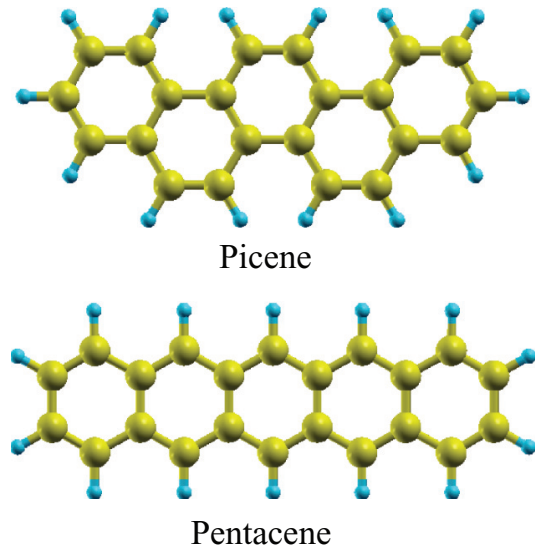

FIG. 1. (Color online) Molecular units of picene and pentacene.

to the band dispersion. ${ }^{16}$ Since $\hat{H}_{\mathrm{CT}-\mathrm{FR}}$ is responsible for scattering processes of an electron (or a hole) from site to site, it gives rise to a mixing between FR and CT states.

In the first stage, we neglect $\hat{H}_{\text {CT-FR }}$ by setting the band dispersion to 0 . The two Hamiltonians $\hat{H}_{\mathrm{FR}}$ and $\hat{H}_{\mathrm{CT}}$ are thus completely decoupled. In pentacene the lowest excited state involves mainly bands deriving from HOMO-LUMO molecular levels. Considering only these bands, the excitonic Hamiltonian simplifies further, becoming diagonal in the band indexes. Excitons in this case can be described using a simple two-level tight-binding model with two molecules in the unit cell. ${ }^{16}$ The eigenstates of the excitonic Hamiltonian consist of pure FR and CT states which can be symmetric or antisymmetric $( \pm)$ with respect to the exchange of an e-h pair between two inequivalent molecules. The energy of FR excitons is

$$
E_{\mathrm{ex}}^{\mathrm{FR} \pm}(\mathbf{q})=\Delta \epsilon-\mathcal{W}+\mathcal{I}(\mathbf{q}) \pm|\mathcal{J}(\mathbf{q})| .
$$

Here $\Delta \epsilon$ is the GW HOMO-LUMO gap, $\mathcal{W}$ is the on-site term of the direct e-h interaction $W$, and $\mathcal{I}$ and $\mathcal{J}$ are the excitation transfer integrals ${ }^{17}$ stemming from the exchange e-h interaction $\bar{v}$ and are related to scattering processes of an e-h pair between two equivalent and inequivalent molecules, respectively. On the other hand, the energy of CT excitons is given by

$$
E_{\mathrm{ex}}^{\mathrm{CT} \pm}(\mathbf{q})=\Delta \epsilon-\tilde{\mathcal{W}},
$$

where $\tilde{\mathcal{W}}$ are the attractive intersite contributions to the direct e-h interaction $W$.

In order to confirm the validity of this simple model description, we solve the $a b$ initio BSE in pentacene, ${ }^{18}$ considering only transitions between HOMO-LUMO bands in which we artificially set the dispersion to 0 . We relax these constraints later. We calculate the optical absorption spectrum, given by the imaginary part of the dielectric function $\epsilon_{2}(\omega)$ in the $\mathbf{q} \rightarrow 0$ limit. In the absence of e-h interactions $[\bar{v}=W=0$ in Eq. (1)], the spectrum has only a single peak located at the HOMO-LUMO gap $\Delta \epsilon$ [see Fig. 2(a)]. In a molecular picture this means that all the e-h excitations become degenerate, and for a given position of the hole, the electron is delocalized everywhere. The effect of the repulsive exchange e-h interaction $\bar{v}$, when $W=0$, is to shift the FR states at higher energies and to remove the degeneracy between symmetric

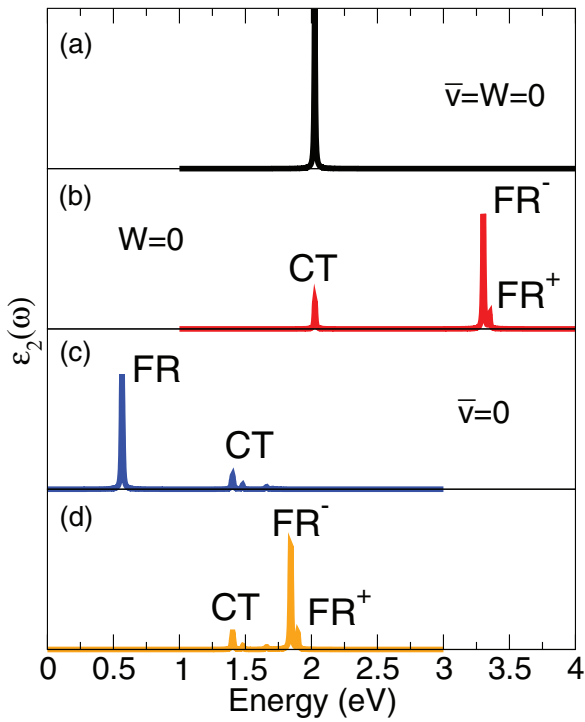

FIG. 2. (Color online) Absorption spectrum of pentacene (along $\left.a^{*}\right)$ where only transitions between HOMO-LUMO bands with zero dispersion are considered. The BSE is solved by (a) neglecting both the direct and the exchange e-h interactions $(\bar{v}=W=0)$; (b) including only the exchange e-h interaction $(W=0)$; (c) including only the direct e-h interaction $(\bar{v}=0)$; (d) and including both the direct and the exchange e-h interactions $W$ and $\bar{v}$.

and antisymmetric FR states [see Fig. 2(b)], i.e., to induce a Davydov splitting. In particular, we see that along $a^{*}$ the more intense $\mathrm{FR}^{-}$peak is located at a lower energy than $\mathrm{FR}^{+11}$. On the contrary, CT states do not feel the exchange e-h interaction [see Eq. (3)] and their energy remains unchanged [compare Figs. 2(a) and 2(b)]. If instead we take into account only the direct e-h interaction $W$ with $\bar{v}=0$ in Eq. (1), all the peaks are red-shifted with respect to the noninteracting case [compare Figs. 2(a) and 2(c)]. Since the on-site $\mathcal{W}$ is always larger than $\tilde{\mathcal{W}}$, the FR exciton has a lower energy than the CT excitons [see Fig. 2(c)]. Moreover, the CT excitons are no longer degenerate: in fact the direct e-h interaction $W$ depends on the e-h separation and thus different CT excitons are determined by different intersite matrix elements $\tilde{\mathcal{W}}$. On the contrary, since $\bar{v}=0$ the degeneracy between symmetric and antisymmetric states is preserved. Finally, we take into account both $W$ and $\bar{v}$ [see Fig. 2(d)]. The exchange e-h interaction $\bar{v}$ is felt only by the FR exciton, hence the energy difference between FR and CT states is reduced. In pentacene the exchange e-h interaction is so strong that when $\bar{v}$ is added to $W$, both symmetric and antisymmetric FR excitons skip above the lowest CT exciton [compare Figs. 2(c) and 2(d)]. As a consequence, in pentacene the lowest-energy excited state in the absence of hopping is a pure CT exciton. This does not happen in picene, for instance, where the exchange e-h interaction is not large enough to compensate for the effect of the direct on-site $\mathcal{W}$ and the lowest-energy excited state is a pure FR exciton.

In the tight-binding picture the exciton dispersion is caused by the scattering of the e-h pair from site to site. In general, scattering processes are induced by hopping through the interaction of an electron (or a hole) with the crystal field or through the effective e-h interaction. However, when the 

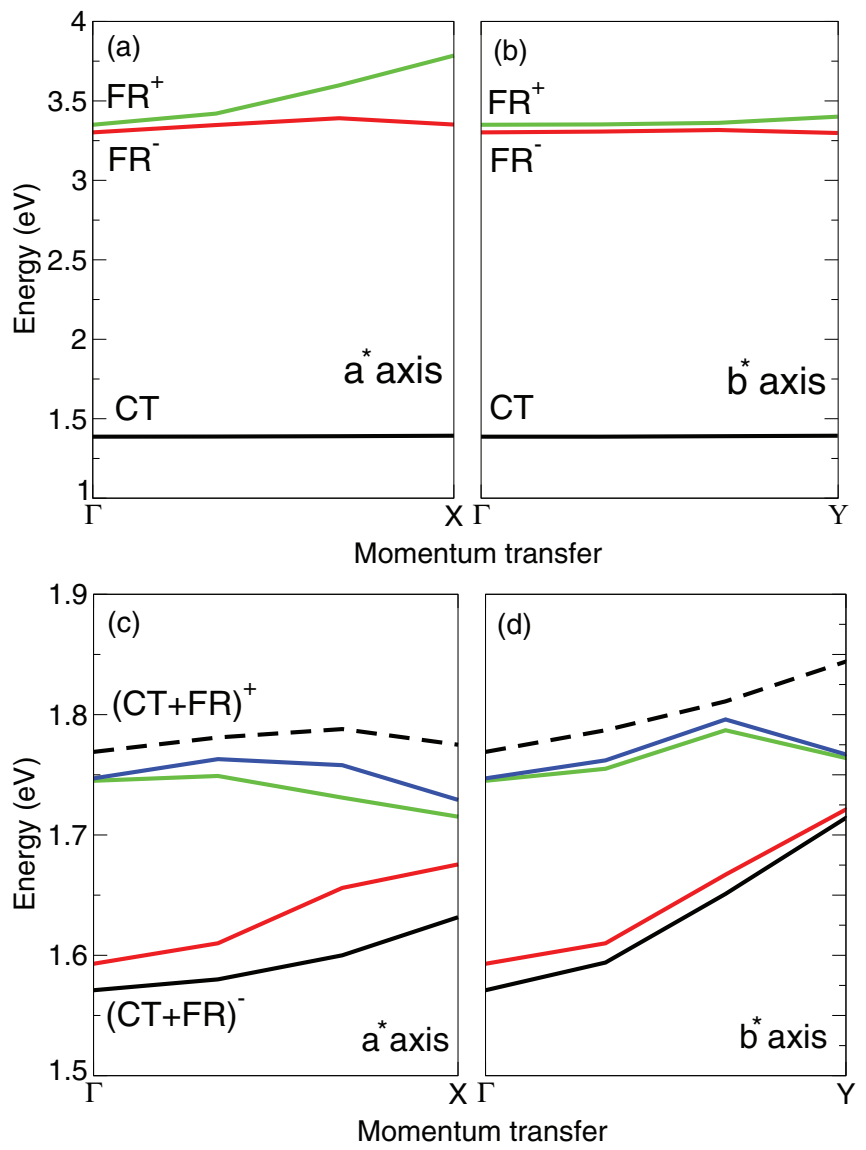

FIG. 3. (Color online) Exciton dispersion in pentacene: (a, b) without hopping and (c, d) including the full band dispersion. Solid and dashed black lines in (c) and (d) refer to excitons related to the coupling between the FR and the lowest CT states. Red, green, and blue lines are other excitons related to the coupling between the FR and the higher-energy CT states.

overlap between wave functions localized on different sites is negligible, the direct $\mathrm{e}-\mathrm{h}$ interaction $W$ cannot induce site-to-site scattering processes. Indeed in Eqs. (2) and (3) both $\mathcal{W}$ and $\tilde{\mathcal{W}}$ are independent of the momentum transfer $\mathbf{q}$. Therefore, in the absence of hopping the exciton dispersion is set only by the exchange e-h interaction $\bar{v}$. This is confirmed by solving the BSE in pentacene with zero hopping as a function of the momentum transfer $\mathbf{q}$ [see Figs. 3(a) and 3(b)]. Since they are not affected by the exchange e-h interaction $\bar{v}$, pure CT excitons do not disperse. On the contrary, both symmetric and antisymmetric FR excitons have a finite dispersion. Therefore the exciton dispersion provides an immediate way to distinguish FR from CT excitons. In particular, for $\mathbf{q}$ along the reciprocal-lattice axis $a^{*}$ the $\mathrm{FR}^{+}$state has an exciton bandwidth of $\sim 0.4 \mathrm{eV}$, which is $\sim 0.1 \mathrm{eV}$ for the $\mathrm{FR}^{-}$state [see Fig. 3(a)]. This suggests that the exchange e-h processes involving equivalent and inequivalent molecules compensate each other in the antisymmetric state, $\mathcal{I}(\mathbf{q})$ and $\mathcal{J}(\mathbf{q})$ having opposite signs for $\mathrm{FR}^{-}$excitons [see Eq. (2)]. On the other hand, for $\mathbf{q}$ along $b^{*}$ [see Fig. 3(b)] the dispersion is negligible.

To investigate the effects of hopping of free electrons and holes, we relax the constraint of flat bands and we solve the
BSE including the full dispersion of the HOMO-LUMO bands. First, through its $\mathbf{q}$ dependence, the hopping induces a finite dispersion of CT states [which otherwise is 0; see Figs. 3(a) and 3(b)] and modifies the intrinsic dispersion of FR excitons given by $\mathcal{I}(\mathbf{q})$ and $\mathcal{J}(\mathbf{q})$ [see Eq. (2)]. Moreover, switching on the hopping induces a mixing of FR and CT states (the coupling term $\hat{H}_{\text {CT-FR }}$ is no longer 0 ): in a real material the excited states are always a mixture of the two excitonic solutions. The hopping hence removes the degeneracy between symmetric and antisymmetric CT states, giving rise to two mixed excitons, with symmetric $(\mathrm{CT}+\mathrm{FR})^{+}$and antisymmetric $(\mathrm{CT}+\mathrm{FR})^{-}$ character. The mixing between $\mathrm{CT}$ and FR excitons is stronger when the hopping is larger and when the energy difference between pure CT and FR states is lower. The e-h exchange interaction, through the terms $\mathcal{I}(\mathbf{q})$ and $\mathcal{J}(\mathbf{q})$ in Eq. (2), changes the energy difference between pure FR and CT excitons as a function of q. In turn, this variation of their separation modulates the mixing effect due to hopping (which is larger when the excitons get closer in energy). We thus see that the exchange e-h interaction, through hopping, also has an (indirect) effect on CT excitons.

Therefore, from this general analysis we can conclude that the exciton dispersion in molecular crystals arises from the interplay between hopping and the exchange e-h interaction. The $a b$ initio BSE results confirm these conclusions. We find [see Figs. 3(c) and 3(d)] that the lowest exciton in pentacene, at $1.55 \mathrm{eV}$ at $\mathbf{q}=0$, is a $(\mathrm{CT}+\mathrm{FR})^{-}$state. It is related to the lowest CT exciton in Figs. 3(a) and 3(b). The corresponding symmetric $(\mathrm{CT}+\mathrm{FR})^{+}$state is at $1.76 \mathrm{eV}$ at $\mathbf{q}=0$, giving a Davydov splitting of $0.2 \mathrm{eV}$. Between these two excitons, other combinations of FR and CT excitons appear. The width of the dispersion of these $(\mathrm{CT}+\mathrm{FR})^{ \pm}$states varies between 0.05 and $0.20 \mathrm{eV}$, which is rightly the order of magnitude of the HOMO-LUMO bandwidths. In particular, the exciton dispersion is larger along the $b^{*}$ axis [see Fig. 3(d)], where the first exciton has a bandwidth of $0.14 \mathrm{eV}$, which is about twice as large as the dispersion along $a^{*}$ [see Fig. 3(c)]. This difference is an indirect effect of the exchange e-h interaction. Because of it, along $a^{*}$ FR excitons have an intrinsic finite dispersion, which is instead negligible along $b^{*}$ [see Figs. 3(a) and 3(b)]. Therefore, as $\mathbf{q}$ increases, FR and CT get farther apart along $a^{*}$, reducing their coupling induced by hopping, while this does not occur along $b^{*}$.

Finally, in Figs. 4(a) and 4(b) we report the spectra of $\epsilon_{2}$ as a function of $\mathbf{q}$ for pentacene, solving the BSE for a converged set of bands (so we relax the last constraint about using only HOMO and LUMO bands). For the lowest-energy peaks we find the same results as obtained by considering only the HOMO-LUMO bands [see Figs. 3(c) and 3(d)], confirming that the HOMO-LUMO bands make by far the most important contribution to these excitons. The lowest $(\mathrm{CT}+\mathrm{FR})^{-}$state is visible for a polarization along the $a^{*}$ axis, while its $(\mathrm{CT}+\mathrm{FR})^{+}$counterpart is visible only along the $b^{*}$ axis. Both excitons remain visible up to the $\Gamma$ point of the second Brillouin zone, where their oscillator strengths drop to 0 and the spectral weight is transferred to higher-energy $(\mathrm{CT}+\mathrm{FR})$ states. Interestingly, at high momentum transfer along the $b^{*}$ axis a new peak appears at an energy lower than that of the $(\mathrm{CT}+\mathrm{FR})^{+}$state that determines the onset at $\mathbf{q}=0$. This new peak corresponds to the lowest $(\mathrm{CT}+\mathrm{FR})^{-}$exciton, 


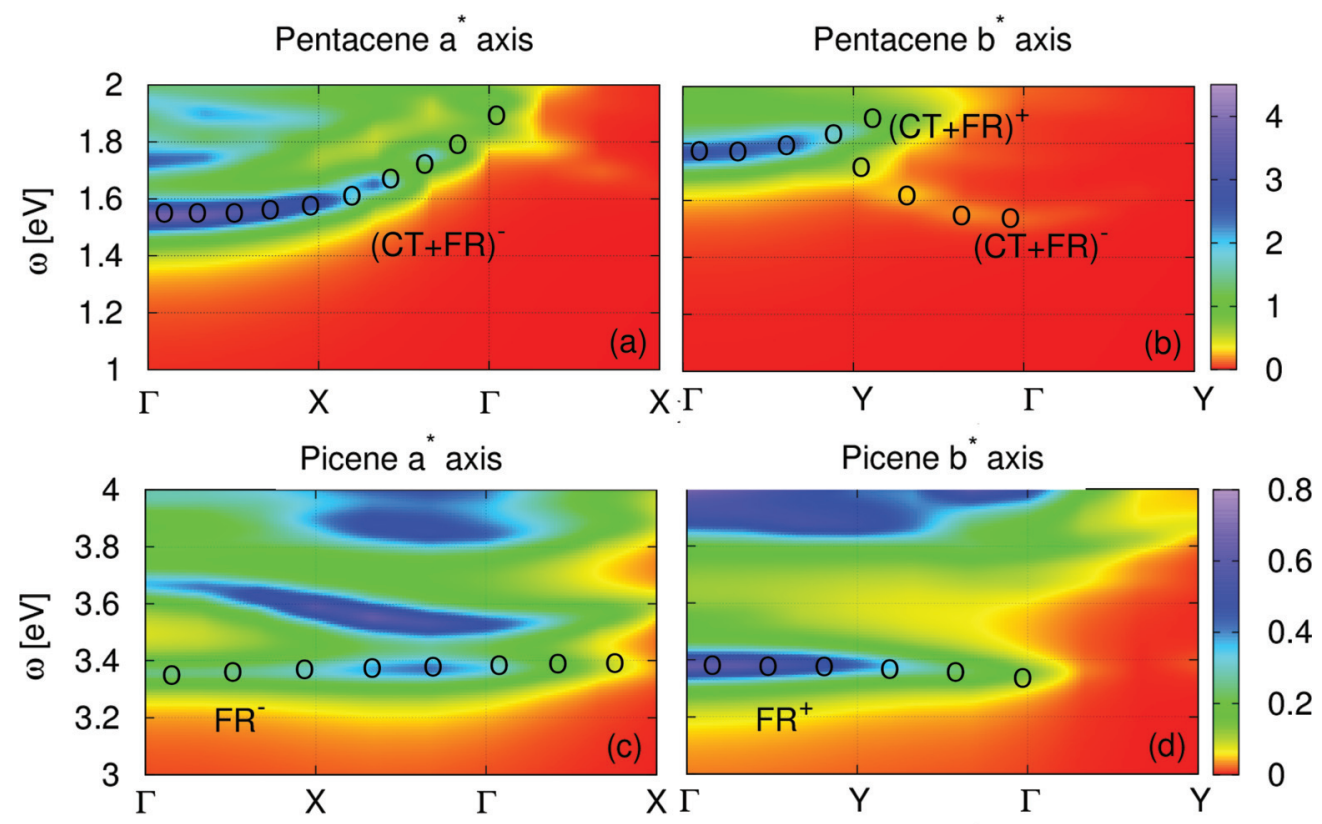

FIG. 4. (Color online) Map of the imaginary part of the macroscopic dielectric function $\epsilon_{2}$ evaluated as a function of the energy (vertical axis) and momentum transfer (horizontal axis) along (a) the $a^{*}$ axis and (b) the $b^{*}$ axis of pentacene and along (c) the $a^{*}$ axis and (d) the $b^{*}$ axis of picene. Black circles are guides for the eye for the lowest-energy excitons.

which is dipole forbidden at low momentum transfer along the $b^{*}$ axis but becomes visible at larger $\mathbf{q}$.

We can now compare pentacene with picene [see Figs. 4(c) and 4(d)], in which the lowest excited states are localized FR excitons. In particular, at $\mathbf{q}=0$ the symmetric and antisymmetric $\mathrm{FR}^{+}$and $\mathrm{FR}^{-}$states are visible along the $b^{*}$ and $a^{*}$ axis, respectively, with a Davydov splitting of about $0.02 \mathrm{eV}$, which is one order of magnitude smaller than in pentacene. These lowest excitons, in contrast to pentacene, involve the contributions of several bands besides the HOMO-LUMO transitions. The mixing of HOMO-LUMO transitions with higher-energy excitations makes the contributions from the direct e-h interaction $W$ larger, while it does not affect the kinetic term in Eq. (1). As a result, the exciton binding energy is also increased, giving rise to a strongly localized FR exciton, well separated in energy from the CT ones. As a consequence, the mixing with higher-energy $\mathrm{CT}$ excitons is negligible in picene. The lowest excited state thus preserves its intrinsic FR character and its dispersion is set by the exchange e-h interaction only. In Figs. 4(c) and 4(d) we see that for small q belonging to the first Brillouin zone, the $\mathrm{FR}^{-}$exciton has a positive dispersion, while the $\mathrm{FR}^{+}$state has a negative dispersion. For both excitons the bandwidth is about $0.02 \mathrm{eV}$, which is one order of magnitude smaller than in pentacene, suggesting that in the two systems the mechanism of the exciton dispersion is completely different.

The traditional interpretation of electroabsorption experiments $^{26,27}$ suggests that the lowest excited state in pentacene is an FR exciton, in seeming contrast with our analysis. In fact, the electroabsorption signal shows different behavior for FR and $\mathrm{CT}$ states. Its shape thus identifies unequivocally the exciton character when this is a pure FR or CT state. However, the interpretation of the electroabsorption spectra is rather complicated in real materials, where the excitons are always a mixture of the two configurations. On the other hand, our results are in good quantitative agreement with recent electron energy-loss spectroscopy (EELS) experiments. ${ }^{28,29}$ While an exciton band structure with bandwidths of about $100 \mathrm{meV}$ has been observed for pentacene, picene has not shown a measurable dispersion for $\mathbf{q}$ belonging to the $a^{*} b^{*}$ plane. The present work thus provides the tools for interpretation of these recent experimental results also.

In conclusion, by combining ab initio many-body BSE calculations for picene and pentacene with a simple model interpretation, we have drawn a general picture of the exciton dispersion in molecular crystals. We have shown that the exciton dispersion is the result of the interplay between two mechanisms: electron and hole hopping and the electronhole exchange interaction. The investigation of the exciton dispersion provides valuable insights into the nature of the excitons, allowing one to tell the difference between localized FR excitons and delocalized CT excitons. This analysis is of general validity and can be exploited to describe other strongly correlated insulators with localized electronic wave functions.

The authors acknowledge financial support from the European Research Council Advanced Grant DYNamo (ERC2010-AdG-267374), Spanish grants (2010-21282-C02-01 and PIB2010US-00652), Grupos Consolidados UPV/EHU del Gobierno Vasco (IT578-13), European Commission projects CRONOS (Grant No. 280879-2 CRONOS CP-FP7), and the Maison de la simulation for technical support. Computational time was granted by GENCI (Project No. 544) and by the CNANO-SIMULEE-2009 project. This work was carried out under the HPC-EUROPA2 project, with the support of the European Community-Research Infrastructure Action of the FP7. 
*pierluigi.cudazzo@ehu.es

${ }^{1}$ L. V. Butov et al., Nature (London) 417, 47 (2002).

${ }^{2}$ D. W. Snoke et al., Nature (London) 418, 754 (2002).

${ }^{3}$ J. Kasprzak et al., Nature (London) 443, 409 (2006).

${ }^{4}$ P. Abbamonte et al., Proc. Natl. Acad. Sci. USA 105, 12159 (2008).

${ }^{5}$ K. Yang et al., Phys. Rev. Lett. 98, 036404 (2007).

${ }^{6} \mathrm{~W}$. Schülke, Electron Dynamics by Inelastic X-Ray Scattering (Oxford University Press, New York, 2007).

${ }^{7}$ F. J. García de Abajo, Rev. Mod. Phys. 82, 209 (2010).

${ }^{8}$ S. Albrecht, L. Reining, R. Del Sole, and G. Onida, Phys. Rev. Lett. 80, 4510 (1998); L. X. Benedict, E. L. Shirley, and R. B. Bohn, ibid. 80, 4514 (1998); M. Rohlfing and S. G. Louie, ibid. 81, 2312 (1998).

${ }^{9}$ G. Onida, L. Reining, and A. Rubio, Rev. Mod. Phys. 74, 601 (2002).

${ }^{10}$ A. Davydov, Theory of Molecular Excitons (McGraw-Hill, New York, 1962).

${ }^{11}$ V. M. Agranovich, Excitations in Organic Solids (Oxford University Press, New York, 2008).

${ }^{12}$ M. Gatti and F. Sottile Phys. Rev. B 88155113 (2013).

${ }^{13}$ W. A. Caliebe, J. A. Soininen, E. L. Shirley, C.-C. Kao, and K. Hämäläinen, Phys. Rev. Lett. 84, 3907 (2000).

${ }^{14}$ A. Marini, R. Del Sole, and A. Rubio, Phys. Rev. Lett. 91, 256402 (2003).

${ }^{15}$ L. Hedin, Phys. Rev. 139, A796 (1965).

${ }^{16}$ P. Cudazzo, M. Gatti, and A. Rubio, Phys. Rev. B 86, 195307 (2012).

${ }^{17}$ F. Bassani and G. Pastori Parravicini, Electronic States and Optical Transitions in Solids (Pergamon Press, Oxford, 1975).
${ }^{18}$ For picene and pentacene we have used the experimental crystal structures of Refs. 19 and 20. In both cases, converged BSE spectra have been obtained with a $6 \times 6 \times 4$ grid of $\mathbf{k}$ points, 53 occupied and 29 empty bands. The coupling between resonant and antiresonant transitions, beyond the Tamm-Dancoff approximation adopted here, does not qualitatively modify our conclusions regarding the dispersion of the lowest-energy excitons and their nature. See also Ref. 21. Ground-state and $G W$ band-structure calculations follow those in Refs. 16, 22, and 23 and were performed with the ABINIT ${ }^{24}$ code. For the BSE at finite $\mathbf{q}$ we used the EXC code. ${ }^{25}$

${ }^{19}$ A. De, R. Ghosh, S. Roychowdhury, and P. Roychowdhury, Acta Crystallogr. C 41, 907 (1985).

${ }^{20}$ O. D. Jurchescu, Ph.D. thesis, Rijksuniversiteit Groningen (2006); http://dissertations.ub.rug.nl/faculties/science/2006/o.d.jurchescu.

${ }^{21}$ C. Ambrosch-Draxl, D. Nabok, P. Puschnig, and Ch. Meisenbichler, New J. Phys. 11, 125010 (2009).

${ }^{22}$ F. Roth, M. Gatti, P. Cudazzo, M. Grobosch, B. Mahns, B. Büchner, A. Rubio, and M. Knupfer, New J. Phys. 12, 103036 (2010).

${ }^{23}$ P. Cudazzo, M. Gatti, F. Roth, B. Mahns, M. Knupfer, and A. Rubio, Phys. Rev. B 84, 155118 (2011).

${ }^{24}$ X. Gonze et al., Z. Kristallogr. 220, 558 (2005).

${ }^{25}$ See http://www.bethe-salpeter.org/.

${ }^{26}$ L. Sebastian, G. Weiser, and H. Bässler, Chem. Phys. 61, 125 (1981).

${ }^{27}$ L. Sebastian, G. Weiser, G. Peter, and H. Bässler, Chem. Phys. 75, 103 (1983).

${ }^{28}$ F. Roth, B. Mahns, B. Büchner, and M. Knupfer, Phys. Rev. B 83, 165436 (2011)

${ }^{29}$ F. Roth, R. Schuster, A. König, M. Knupfer, and H. Berger, J. Chem. Phys. 136, 204708 (2012). 